\title{
Commentary on Jansen's Paper
}

\author{
Melvin L. Morse, M.D. \\ University of Washington
}

\begin{abstract}
Karl Jansen raises a fundamental and exciting question: Is humankind's consciousness the result of neuronal function, or are there extracerebral aspects as well? While his neurotransmitter model of near-death experiences (NDEs) is well described, I find his supporting evidence weak. Methodological differences between studies of ketamine hallucinations and near-death experiences (NDEs) raise doubts about how similar those experiences are phenomenologically. While Jansen's model has electrifying implications, the data required to support his conclusions do not yet exist.
\end{abstract}

This long-awaited article by Karl Jansen is a follow-up to his intriguing letter to the British Medical Journal in 1989. His main argument proceeds from assumptions that cannot properly be addressed, given the current state of the literature. He could not raise a more fundamental and exciting question, which is: Is humankind's consciousness the result of neuronal function, or are there extracerebral aspects as well? It is exciting to see a scientist tackle that issue, using clinical and experimental data to address it. Most previous discussions are speculative and philosophical.

Here comes Jansen, who has the courage to say that there is overwhelming evidence that mind results from neuronal activity; but it is frustrating to realize that most of the references he cites are suspect. This then is the challenge for near-death research. We must start to generate data worthy of the questions we are attempting to answer. Theorists like Jansen need clinical researchers like Raymond Moody and Kenneth Ring; this is the beginning of a dialogue that will blossom into something truly valuable.

Melvin L. Morse, M.D., is Associate Professor of Pediatrics at the University of Washington. Reprint requests should be addressed to Dr. Morse at the Valley Children's Clinic, 4011 Talbot Road South, Suite 220, Renton, WA 98055. 
Articles such as Jansen's I believe point the way to the day that funding will be available for large scale prospective studies that will resolve so many of the fundamental issues that Jansen grapples with. For example, he attempts to compare the phenomenology of ketamine hallucinations and near-death experiences (NDEs), yet the studies cited are in no way comparable. This is not Jansen's fault: he is citing the literature as it is. Yet his article highlights the importance of designing studies that will permit proper data that would be helpful in such an article.

Many major scientific advances have resulted from this sort of dynamic. For example, early studies of the histology and function of the hippocampus were confusing and contradictory, often because of differences in experimental technique. Theoretical pressures forced researchers to reanalyze old data and generate new experimental designs that corrected flaws in earlier research, resulting in our current understanding of hippocampal function.

One argument against NDEs being generated by neurotransmitters at the point of death is that it is difficult to understand the evolutionary pressures that would result in the evolution of NDEs occurring to comatose dying persons. Jansen's theory is that neuroprotective agents released by the dying brain also generate an expanded sense of awareness and consciousness. Although he does not specifically articulate the benefits of dying people having NDEs, clearly it would allow for a sense of calm, alertness, and peace, which could result in life-saving action. I once rescued a horse from barbed wire that was threatening to break the horse's leg. Perhaps a similar phenomenon in that horse occurred, as the horse instinctively lay still, did not struggle, and allowed us to rescue it.

Jansen's paper is at its best when it sticks to what can be referenced properly in the literature; but its weaknesses significantly detract from the main point he is making. His neurotransmitter model by itself is well-referenced and described. Its implications are obvious and electrifying. It could trigger tremendous debate, and furthermore, has the power to inspire considerable experimental and clinical research. Jansen further attempts to incorporate this ketamine model into a comprehensive model of the neurobiology of NDEs.

My specific criticisms of his paper relate to his citations of supportive evidence. He states that there is overwhelming evidence that 
mind results from neuronal activity, but does not provide substantiating references. He states that all the features of the classic NDE can be produced by ketamine. Unfortunately, we have no standard tool to define the NDE or its classic features. Memories of cardiac arrest survivors and recreational ketamine users are hard to compare from descriptions in the literature, all of which were collected in different ways from examiners looking for different phenomenology and having different biases. Jansen further states that lysergic acid diethylamide (LSD) does not cause NDE-like experiences, again without convincing references. The real problem is that no really systematic study of this has been done, although articles delineating schizophrenic hallucinations from NDEs exist.

Even given this weakness in the literature, Jansen needs to define more clearly and justify his contention that NDEs and ketamine effects are indistinguishable from a clinical point of view. After all, his entire premise is based on this point. Many of the references he cites are not primary sources, but are secondary references themselves. Quoting speculations by Ronald Siegel $(1980,1981)$, for example, does not properly support his own theory. He does not cite what little work has been done in this area, such as Bruce Greyson's NDE Scale (1983), Kenneth Ring's Weighted Core Experience Index (1980), or the diagnostic work of Brian Bates and Adrian Stanley (1985).

Jansen does not cite enough primary sources of descriptions of NDEs and how those data were collected. He does not describe ketamine hallucinations in detail, or how those descriptions were collected. For example, Scott Rogo (1984) described ketamine hallucinations as having a paranoid flavor to them, and ultimately being dissimilar to NDEs. Although ketamine hallucinations and NDEs might have some areas of overlap, can they be distinguished from one another nine out of ten times, or only one out of a thousand times? Jansen does not address these issues at all.

Jansen's model has the potential to be an enormously important one, one that will be debated for years to come. It could inspire relevant research, from positron emission tomography scans of ketamine users to clinical studies of the phenomena experienced by users of LSD and ketamine and by NDErs. In its present form, there are too many ideas and speculations mixed together. It needs better defini- 
tion of what is opinion, philosophical speculations, and a better description of the empirical evidence in the literature.

\section{References}

Bates, B. C., and Stanley, A. (1985). The epidemiology and differential diagnosis of near-death experience. American Journal of Orthopsychiatry, 55, 542-549.

Greyson, B. (1983). The Near-Death Experience Scale: Construction, reliability, and validity. Journal of Nervous and Mental Disease, 171, 369-375.

Jansen, K. L. R. (1989). Near-death experience and the NMDA receptor [Letter]. British Medical Journal, 298, 1708.

Ring, K. (1980). Life at death: A scientific investigation of the near-death experience. New York, NY: Coward, McCann and Geoghegan.

Rogo, D. S. (1984). Ketamine and the near-death experience. Anabiosis: The Journal of Near-Death Studies, 4, 87-96.

Siegel, R. K. (1980). The psychology of life after death. American Psychologist, 35, 911-950.

Siegel, R. K (1981, January). Accounting for "afterlife" experiences. Psychology Today, pp. 65-75. 